Eur J Clin Chem Clin Biochem

1995; 33:263-265

(c) 1995 Walter de Gruyter \& Co.

Berlin - New York

\title{
The Reticulocyte Count and Its Subfractions in Smoking and Non-Smoking Pregnant Women
}

\author{
By Patricia E. A. M. Mercelina-Roumans ${ }^{1}$, Johannes M. H. Ubachs ${ }^{1}$ and Jan W. J. van Wersch ${ }^{2}$
}

1 Department of Obstetrics and Gynaecology

2 Department of Haematology

De Wever Hospital, Heerlen, The Netherlands

(Received November 11, 1994/February 12, 1995)

Summary: Our objective was to compare the reticulocyte count and its subfractions in smoking and non-smoking women at different stages of normal pregnancy.

Study design: In 247 non-smoking and 123 smoking healthy pregnant women the reticulocyte count and its subfractions were compared at four different stages of pregnancy: $0-10,11-20,21-30$ and $31-40$ weeks. Exclusion criteria were a diastolic blood pressure $\geq 90 \mathrm{mmHg}$, an endocrine disease or a coagulation disorder. Women in the smokers group smoked more than 4 cigarettes a day. Non-smokers were defined as women reporting no smoking at all. Blood samples were run on the Sysmex R-3000 reticulocyte counter.

Results: The absolute reticulocyte count was lower in the smoking group throughout pregnancy, but this was only significant in the last ten weeks of gestation $\left(71.9 \times 10^{9} / 1\right.$ versus $\left.78.8 \times 10^{9} / 1\right)$. There was no difference between the low fluorescence, the medium fluorescence and the high fluorescence proportions in the non-smoking and the smoking group. Both groups behaved similarly during pregnancy; there was a decrease of mature reticulocytes and a significant increase of more immature reticulocytes.

Conclusion: These data show a moderate measurable effect of cigarette smoking on the reticulocyte count and the absence of an effect on the reticulocyte subsets.

\section{Introduction}

Reticulocyte counting allows the direct measurement of the activity of erythropoiesis in the bone marrow. Manual reticulocyte counting has been reported as problematic $(1-5)$. To improve the reliability of reticulocyte counting, several automated methods and instruments have been introduced (6-8). The Sysmex R-3000 was used in this study. This instrument is able to provide precise and statistically reliable reticulocyte counts (9). Furthermore, it estimates the maturity of a reticulocyte by measuring the fluorescence intensity, a reflection of the RNA content of the cell. Castriota et al. (10) studied the total reticulocyte count and the reticulocyte fluorescence intensity ratios in a normal paediatric population. rate evaluation of bone marrow activity than the total reticulocyte count.

The reticulocyte population can be divided into three groups. They are defined by ratios of the total fluorescence intensity and termed low, medium and high fluorescence ratios (11). As the reticulocytes become older, their fluorescence (RNA content) decreases. The high fluorescence ratio is therefore a reflection of the most immature reticulocyte. These phenomena have not been investigated in pregnant women. The aim of this study was to compare these quantities in smoking and non-smoking females during a normal pregnancy. 
Tab. 1 Basic characteristics of the pregnant women.

\begin{tabular}{lllll}
\hline Groups of patients & $\begin{array}{l}\text { Age } \\
\text { (a) } \\
\text { median } \\
\text { (interquartile range) }\end{array}$ & $\begin{array}{l}\text { Iron } \\
\text { medication }\end{array}$ & Parity & $\begin{array}{l}\text { Cigarettes per day } \\
\text { median } \\
\text { (interquartile range) }\end{array}$ \\
\hline $\begin{array}{l}\text { Non-smokers, normal pregnancy } \\
(\mathrm{n}=247)\end{array}$ & 30 & $20 \%$ & $47.9 \%$ primi & .1 \\
$\begin{array}{l}\text { Smokers, } \\
(\mathrm{n}=123)\end{array}$ & $(27-33)$ & & $52.1 \%$ multi & \\
\hline
\end{tabular}

\section{Materials and Methods}

Two hundred and forty-seven non-smoking and 123 smoking healthy pregnant women were included in the study. The reticulocyte count and subsets were determined at four different stages $(0-10$, $11-20,21-30,31-40$ weeks) of pregnancy. Not all patients were checked in all four stages. Exclusion criteria were a diastolic blood pressure $\geq 90 \mathrm{mmHg}$, an endocrine disease or a coagulation disorder. The duration of the gestation was based on the last menstrual period and an ultrasound determination between 8 and 14 weeks. The number of cigarettes smoked per day was estimated by the patient, in most cases confirmed by her partner. If a women smoked more than 4 cigarettes a day she was called a smoker. The group of women who smoked more than 15 cigarettes a day was too small to subdivide the smokers into an intermediate smoking level group (5-15 cigarettes per day) and a group of heavy smokers ( $>15$ cigarettes per day). Non-smokers were defined as women reporting no smoking at all. The basic characteristics of these patients are given in table 1.

Venous blood samples were collected in EDTA- $\mathrm{K}_{2}$ containing tubes (Sarstedt, Nümbrecht, Germany). Samples were kept at room temperature until analysis. The measurements were performed with the relatively new Sysmex R-3000 reticulocyte counter (Toa Medical Electronics, Kobe, Japan). The coefficients of the inter-assay variation for the reticulocyte count amounted to $6.7 \%$ and $7.6 \%$ at levels of $14 \times 10^{9} / \mathrm{l}$ and $36 \times 10^{9} / \mathrm{l}$, respectively (reference range for women $20-110 \times 10^{9} /$ ). This instrument provides the reticulocyte count as a percentage of the erythrocytes, the absolute reticulocyte count, the red blood cell count and the platelet count as well as a cytogram from which the reticulocytes can be subdivided into low, medium and high fluorescence ratio reticulocytes, in that order indicating three consecutive stages in the maturation of the reticulocytes. Correlations were determined by linear regression analysis with the method of the least squares. The significance of the differences of the median values of the various groups was assessed with the Mann-Whitney-Wilcoxon test.

\section{Results}

Figure 1 gives a comparison of the median values of the absolute reticulocyte count of smoking and non-smoking females in four stages of pregnancy.

The reticulocyte count throughout pregnancy was lower in the smoking group but this was only significant in the $31-40^{\text {th }}$ week of gestation $\left(71.9 \times 10^{9} / 1\right.$ versus 78.8 $\left.\times 10^{9} / \mathrm{l} ; \mathrm{p}<0.014\right)$.

The median low, medium and high fluorescence ratio percentages did not differ significantly between the two groups. The three reticulocyte subfractions behaved similarly in both groups. The low fluorescence ratio de- creased (from 88.5 to $78.6 \%$ in the non-smoking, from 88.3 to $77.4 \%$ in the smoking group), whereas the medium fluorescence ratio (from 10.7 to $18.0 \%$ in the nonsmoking, from 10.8 to $17.4 \%$ in the smoking group) and the high fluorescence ratio (from 1.0 to $3.6 \%$ in the nonsmoking, from 1.1 to $4.4 \%$ in the smoking group) increased significantly from the beginning to the end of gestation.

\section{Discussion}

The erythrocyte count and indices in smoking and nonsmoking pregnant women were studied earlier (12). Smoking pregnant women appeared to have lower erythrocyte counts from the $11^{\text {th }}$ week of gestation onwards. An increase of bone marrow activity and a consequent increase of reticulocytes was to be expected in the same period. In the present study however, smoking pregnant women had a lower reticulocyte count in the last ten weeks of pregnancy, suggesting an absence of increased bone marrow activity.

The reticulocyte subsets did however show a significant increase of bone marrow activity, both in non-smoking and in smoking pregnant women. The low fluorescence

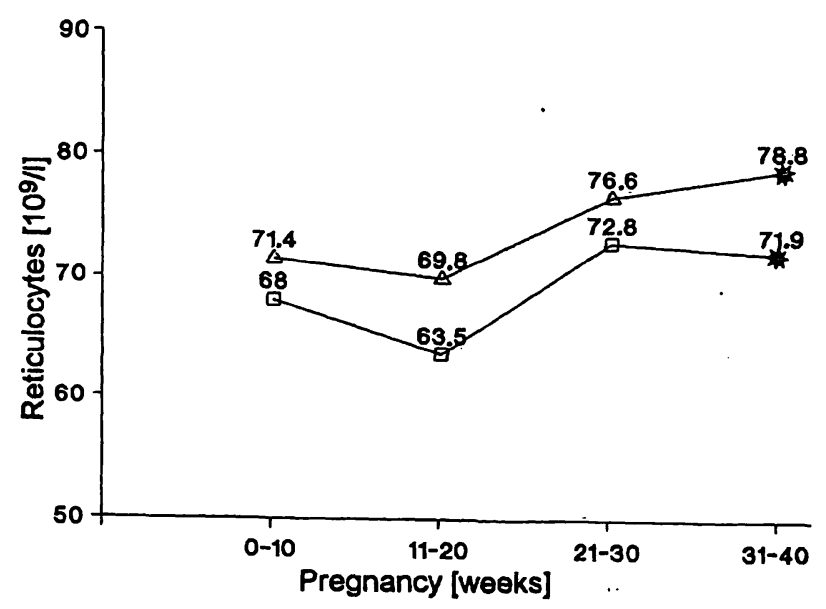

Fig. 1 Comparison of the absolute values of the reticulocyte count of the smoking $(\square)$ and non-smoking $(\Delta)$ females in four stages of pregnancy. Significant difference is indicated by an asterisk (*). 
ratio, indicating more mature reticulocytes, decreased, whereas the medium and high fluorescence ratios, representing the more immature reticulocytes, increased significantly from the beginning to the end of gestation. The relative stability of the reticulocytes and the steady increase of the reticulocyte subsets suggest a rapid decrease of the intra-erythrocytic RNA content in the course of reticulocyte maturation during pregnancy. This process seems to be independent of whether or not the pregnant women smoke. This is remarkable as one would expect a counterregulation of erythropoiesis as a reaction to the decline of erythrocytes due to smoking in pregnancy.

Cigarette smoke may have an effect on the bone marrow and thus affect erythropoiesis. German (13) found an inhibition of erythropoiesis: in individuals who smoked less than five years the reticulocyte maturation rate was reduced, as was the level of circulating red cells. Controversially, the erythropoiesis was normalized in those subjects who smoked more than five years. The author stated that these findings reflect

\section{References}

1. Dessis A, Kurth D. Circulating reticulocytes in normal adults as determined by the new methylene blue method. Am J Clin Pathol 1970; 53:207-11.

2. Gilmer PR, Koepke JA. The reticulocyte, an approach to definition. Am J Clin Pathol 1976; 66:262-7.

3. Greenberg ER, Beck JR. The effects of sample size on reticulocyte counting and stool examination. Arch Pathol Lab Med 1984; 108:396-8.

4. May JA, Sage BH. Spinner films for reticulocyte counts. Am J Med Technol 1976; 42:357-60.

5. Peebles DA, Hochberg A, Clarke TD. Analysis of manual reticulocyte counting. Am J Clin Pathol 1981; 76:713-7.

6. Tanke HJ, Rotharth PH, Vossen JMJJ, Koper GJM, Ploem JS. Flow cytometry of reticulocytes applied to clinical laboratory. Blood 1983; 5:225-36.

7. Tatsumi N, Tsuda I, Kojima K, Niri M, Setoguchi K. An automated reticulocyte counting method: preliminary observations. Med Lab Sci 1989; 46:157-60.

8. Vaighan WP, Hall J, Johnson K, Dougherty C, Peebles DA. Simultaneous reticulocyte and platelet counting on a clinical flow cytometer. Am J Hematol 1985; 18:385-91.

9. Shoustal AM. Evaluation of the Sysmex R-3000 automated reticulocyte analyzer with comparison to the Sysmex R-1000. Sysmex Int J 1992; 2:16-25. different phases of the toxic effects of tobacco smoke on the bone marrow.

However, cigarette smoke may also have a direct effect on the erythrocyte itself. The same investigator found a toxic effect on the red cell. Six to ten years of smoking resulted in toxic lesions of the circulating erythrocytes confirmed by an increase of the number of spherulation altered cells, early onset and a late termination of haemolysis and an increased percentage of perished cells (14). The biological mechanism is unclear.

\section{Conclusion}

The measurable effect of cigarette smoking on the reticulocyte count was moderate, whereas the effect on the low, medium and high fluorescence proportions was absent. These results indicate that no real counterregulation occurs as a reaction to the decline of erythrocytes due to smoking in pregnancy. The mechanisms by which smoking affects the bone marrow or the erythrocyte itself remains unclear.

10. Castriota-Scanderbeg A, Pedrazzi G, Mercadanti M, Stapane I, Butturini A, Izzi G. Normal values of total reticulocytes and reticulocyte subsets in children. Haemotol 1992; 77:363-4.

11. Sato K, Kimuna T, Shigera S, Koyanagi Y, Hiroko H, Sugawara $\mathrm{K}$. The clinical usefulness of reticulocyte classification by fluorescence intensity. Sysmex Int J 1991; 14:29-35.

12. Mercelina-Roumans PEAM, Ubachs JMH, van Wersch JWJ. Erythrocyte count and indices during normal pregnancy of non-smoking and smoking women. Eur J Obst Gynecol Reprod Biol 1994; 57:25-8.

13. German AK. The effect of smoking on the composition of the peripheral blood in normal subjects. Lab-Delo 1991; 10:42-5.

14. German AK. The erythrocyte composition of the peripheral blood in tobacco smokers. Vrach-Delo 1992; 7:40-2.

\author{
Dr. J. W. J. van Wersch \\ Department of Haematology \\ De Wever Hospital \\ P. O. Box 4446 \\ NL-6401 CX Heerlen \\ The Netherlands
}




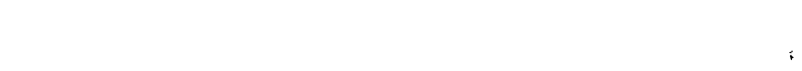

